\title{
Is media search a reliable source when investigating the incidence of sports-related out-of-hospital cardiac arrest?
}

Cecilie B. Isern ${ }^{1,2,4}$, Ingvild B. M. Tjelmeland ${ }^{3}$, Jo Kramer-Johansen ${ }^{1,4}$, Eivind Berge ${ }^{5}$,Hilde Moseby Berge ${ }^{2}$

${ }^{1}$ Norwegian National Advisory Unit on Prehospital Emergency Medicine (NAKOS), Oslo University hospital, ${ }^{2}$ Oslo Sports Trauma Research Center, Norwegian School of Sport Sciences, ${ }^{3}$ Norwegian Cardiac Arrest Registry (NorCAR) at Norwegian National Advisory Unit on Prehospital Emergency Medicine (NAKOS), Oslo University hospital, ${ }^{4}$ Faculty of Medicine, University of Oslo,

${ }^{5}$ Department of Cardiology, Oslo University Hospital

\section{Background}

Media records are a common source when reporting incidence of sports-related out-ofhospital cardiac arrest (OHCA). The incidence of OHCA increases with age, and OHCA in older age groups might be less likely to cause media attention.

\section{Purpose of the study}

Assess the incidence of OHCA related to sport in the Norwegian Cardiac Arrest Registry (NorCAR) depending on age, and compare this with media reports.

\section{Materials and methods}

Results from a systematic search in the media database Retriever was compared with data from NorCAR for the years 2015 and 2016. We divided the population in two age groups: $<40$ and $\geq 40$. We included OHCAs with presumed cardiac aetiology that were classified as sportsrelated (figure 1) from NorCAR.

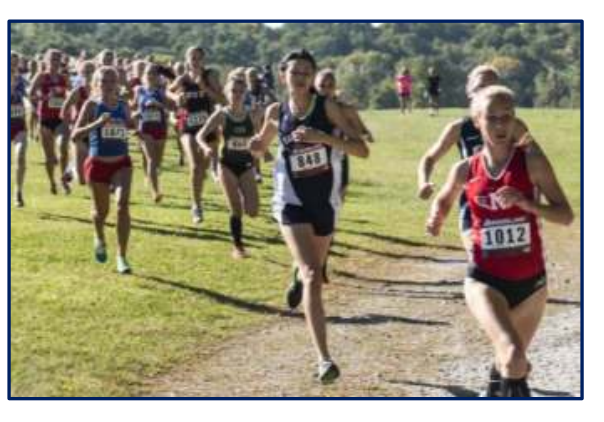
- OHCA during or $<60$ minutes after exercise
OR

- OHCA at an area for "sport or recreational activities".
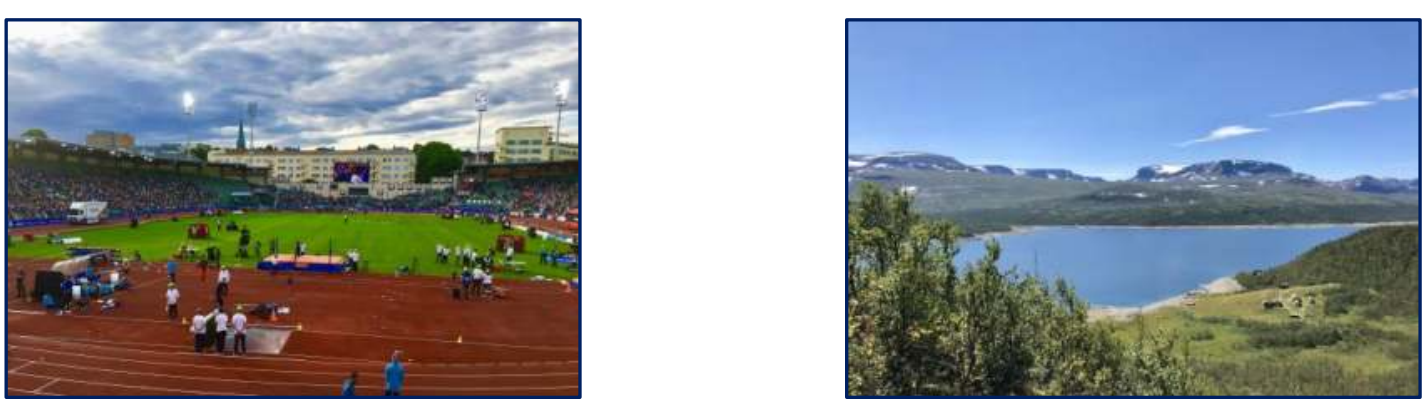

Figure 1. Criteria for classifying OHCA as sports-related in NorCAR.

200

150

100

50

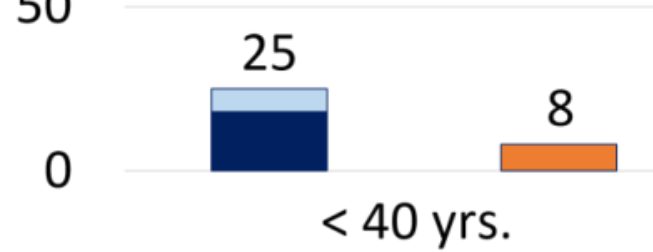
$<40$ yrs.

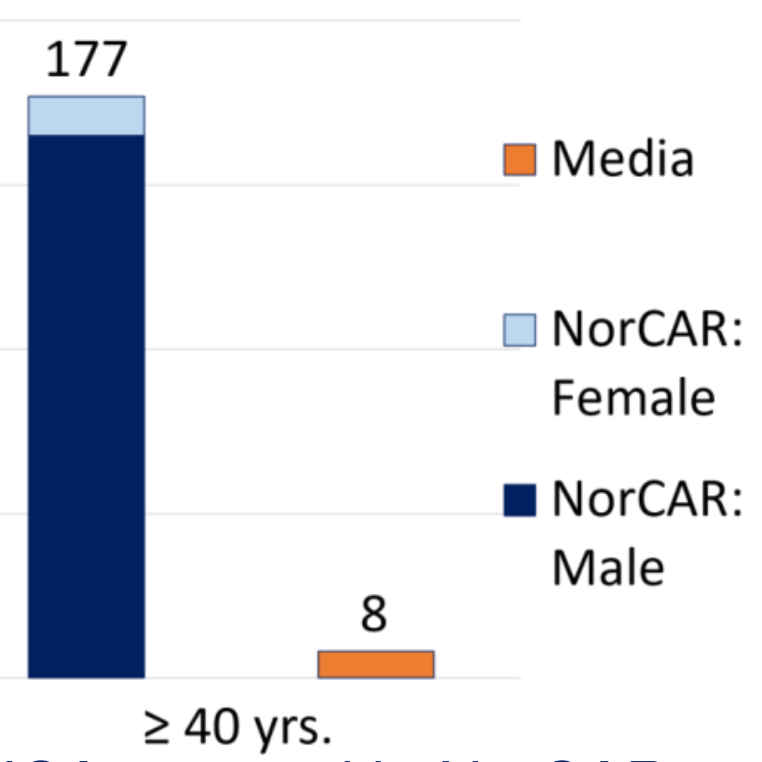

media by age groups.
Results

We identified 4631 events with presumed cardiac aetiology in NorCAR during the 24 months. 202 (4\%) cases matched at least one criterion for sports-related OHCA; $25<40$ years, and $177 \geq 40$ years. Media search resulted in 16 cases; eight in each age group (figure 2), 13 of which were identified in NorCAR and 3 that were not (figure 3).

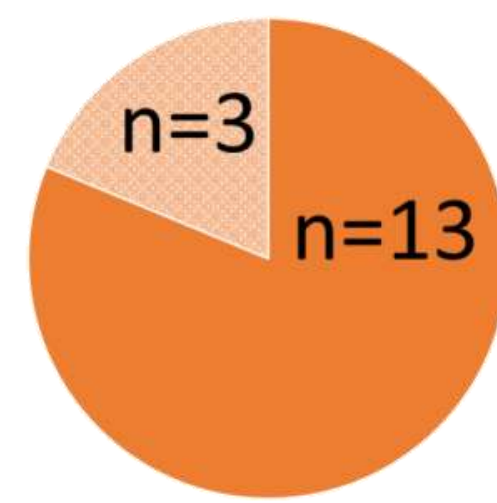

- Sports-related OHCA reported both in media and NorCAR.

Sports-related OHCA identified in media only.

Figure 3. A total of 16 cases of sports-related OHCA were found in media; 13 of these were also identified in NorCAR and 3 were identified in media only.

\section{Conclusion}

Compared to the numbers of OHCA reported to be sports-related in NorCAR, a systematic media search highly underreported the incidence of sports-related OHCA, especially in the older age group ( $\geq 40$ years of age). 